\title{
Effects of Saliva Secretion on the Assessment of Masticatory Performance
}

\author{
Hiroshi Shiga, BEng, DDS, PhD, Yoshinori Kobayashi, DDS, PhD, Ichiro Arakawa, DDS, PhD, \\ Masaoki Yokoyama, DDS, PhD, and Nerihisa Namba, DDS, PhD \\ Department of Partial and Complete Denture, School of Life Dentistry at Tokyo, The Nippon Dental University, \\ Tokyo, Japan
}

\section{Clinical significance}

This study confirms that the saliva secreted during gummy jelly chewing has an influence on the masticatory performance which was obtained by measuring glucose extraction.

\begin{abstract}
Purpose: The purpose of this experiment was to clarify whether the amount of saliva secreted during the chewing gummy jelly affected the assessment of masticatory performance.

Methods: Twenty healthy subjects were asked to chew 2 g of gummy jelly for 10, 15, and 20 seconds and the amounts of glucose extraction and saliva secretion were measured. The changes in both glucose extraction and saliva from 10 to 20 seconds were investigated. Next, the amount of glucose extraction excluding the effect of saliva (extraction B) was divided by the amount of glucose extraction measured (extraction A), and the results for the two chewing times were compared.

Results: The amount of glucose extraction increased proportionally as the chewing time progressed and the change was significant. Although the amount of saliva secretion increased as the chewing time progressed, the change differed from the proportional change in glucose extraction. The amount of saliva secretion was extensive for the first 10 seconds and increased about $0.1 \mathrm{ml}$ every 5 seconds. The ratio of the extraction $\mathrm{B}$ to extraction A was between 1.03 and 1.05, and there was a significant difference between each pair of chewing times. Conclusion: From these results it was concluded that the amount of saliva secretion increased during chewing of gummy jelly and affected the assessment of masticatory performance, and this effect was less than $5 \%$ for chewing of up to 20 seconds.
\end{abstract}

Corresponding to: Dr Hiroshi Shiga

Department of Partial and Complete Denture,

School of Life Dentistry at Tokyo, The Nippon Dental

University,

1-9-20 Fujimi, Chiyoda-ku, Tokyo 102-8159, Japan

Tel: +81-3-3261-5729, Fax: +81-3-3261-8464

E-mail: h-shiga@tokyo.ndu.ac.jp

Received on February 12, 2007 / Accepted on March 21, 2007
Key words: mastication, masticatory performance, gummy jelly, saliva, glucose extraction

\section{Introduction}

Masticatory performance is an important indicator in objectively evaluating masticatory function. The sieve method, ${ }^{1-3}$ whereby a certain quantity of test food is chewed for a certain number of times and then retrieved and measured with sieves of various mesh sizes, is used as the standard method of evaluating masticatory performance. However, because of the drawbacks of this method, such as being complicated and time-consuming, other relatively simple methods using silicone impression materials, ${ }^{4,5}$ bagged almonds, ${ }^{6}$ chewing gum, ${ }^{7-9}$ paraffin wax, ${ }^{10}$ and gummy jelly ${ }^{11,12}$ have been tried in recent years. Of these methods, measuring the glucose extraction from chewing gummy jelly has received a great deal of attention for several reasons: it is easy to use, it is hygienic, the shape and characteristics of gummy jelly can be easily standardized. There was a positive correlation between the masticatory performance as measured by the amount of glucose extraction and the masticatory performance as measured by the sieve method. ${ }^{13}$ However, how the saliva secreted during gummy jelly affects glucose concentration measured after chewing was not known. Therefore, this experiment analyzed the glucose extraction and saliva secretion after chewing of gummy jelly in order to clarify whether the amount of saliva secreted during the chewing of gummy jelly affected the assessment of masticatory performance. 
Table 1 Size, weight, hardness, and ingredients of the gummy-jelly.

\begin{tabular}{llc}
\hline Size $(\mathrm{mm})$ & & \\
\hline Weight $(\mathrm{g})$ & & 2 \\
\hline Hardness $(\mathrm{kgf})$ & & 2.17 \\
\hline Ingredients & Maltose & $40 \%$ \\
& Solbitol & $10 \%$ \\
& Glucose & $5 \%$ \\
& Gelatin & $8 \%$ \\
& Other & $37 \%$ \\
\hline
\end{tabular}

\section{Materials and methods}

\section{Subjects and test food}

Twenty healthy subjects (10 males and $10 \mathrm{fe}^{-}$ males) between 21 and 34 years of age (average, 27.4 years) were selected. The general nature of the study was explained to the subjects and then their informed consent was obtained. None of the subjects had clinical abnormalities in the masticatory system. The following selection criteria were applied: no complaints about occlusion, a full complement of teeth excluding third molars, no major dental restoration, and no orthodontic treatment. The test food was one piece of cylindrical-shaped gummy jelly, $10 \mathrm{~mm}$ in diameter and $10 \mathrm{~mm}$ in height, weighing $2 \mathrm{~g}$. Gummy jelly was prepared according to the ingredients listed in Table 1.

\section{Recording method}

After chewing the gummy jelly on their habitual chewing side for 10,15 , and 20 seconds, subjects were asked to rinse out their mouths with $10 \mathrm{ml}$ distilled water and to spit into a cup with a filter, and the filtrate was collected. The amount of glucose extraction and the amount of saliva secretion were measured. For the amount of glucose extraction, the glucose concentration measured using a portable blood glucose testing device was calibrated by a correction equation ((measured value+35.30)/1.199). 14 The amount of saliva secretion was obtained by subtracting the weight of distilled water and from the weight of the filtrate and gummy jelly.

\section{Method of analysis}

The changes from 10 to 20 seconds were investigated using repeated measures analysis of variance (ANOVA) for both glucose extraction and saliva. Next, the amount of glucose extraction excluding the effects of saliva (extraction B) was

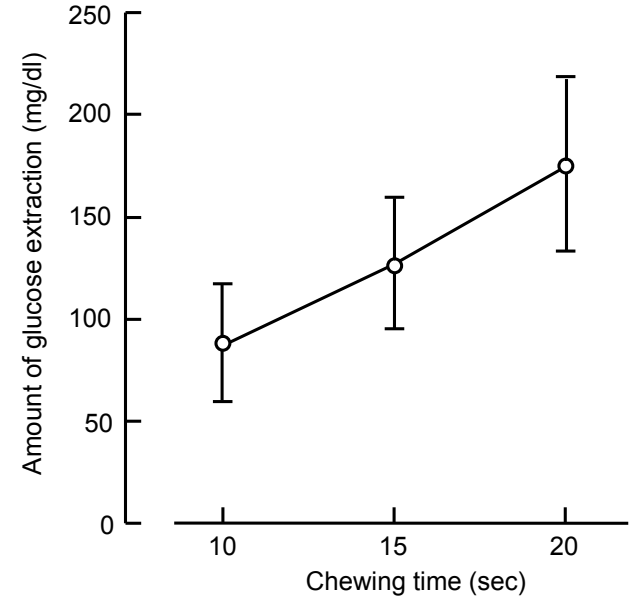

Fig. 1 The average value and standard deviation of the amount of glucose extraction measured (extraction A) for 10 , 15 , and 20 seconds.

divided by the amount of glucose extraction measured (extraction A), and the results for the two chewing times were compared. Extraction B was calculated using the following formula: Extraction $\mathrm{B}=$ Extraction $\mathrm{A} \times$ weight of filtrate/(weight of filtrate-weight of saliva). A comparison was performed using Bonferroni's multiple comparison test. All of the data were analyzed with the statistical software SPSS for Windows, version 10.0J (SPSS, Chicago, IL, USA). $P$ values $<0.05$ were considered to be significant.

\section{Results}

The amounts of glucose extraction for 10, 15, and 20 seconds were $87.6 \mathrm{mg} / \mathrm{dl}, 125.2 \mathrm{mg} / \mathrm{dl}, 175.1$ $\mathrm{mg} / \mathrm{dl}$ respectively (Fig. 1), and increased proportionally as the chewing time progressed; the change was significant $(F=247.0, P<0.01)$. The amounts of saliva secretion for 10,15 , and 20 sec- $^{-}$ onds were $0.28 \mathrm{ml}, 0.36 \mathrm{ml}, 0.46 \mathrm{ml}$ respectively (Fig. 2), and the change was significant ( $F=23.4$, $P<0.01)$. However the change differed from the proportional change in glucose extraction. The amount of saliva secretion was extensive for the first 10 seconds and increased about $0.1 \mathrm{ml}$ every 5 seconds. The ratio of the extraction B to extraction A for 10, 15, and 20 seconds was 1.03, 1.04, and 1.05 respectively (Fig. 4), and there was a significant difference between each pair of chewing times $\left(10 \mathrm{~s}^{-15 \mathrm{~s}}: P=0.048,15 \mathrm{~s}^{-} 20 \mathrm{~s}: P=0.004\right.$, $10 \mathrm{~s}-20 \mathrm{~s}: P<0.001)$. 


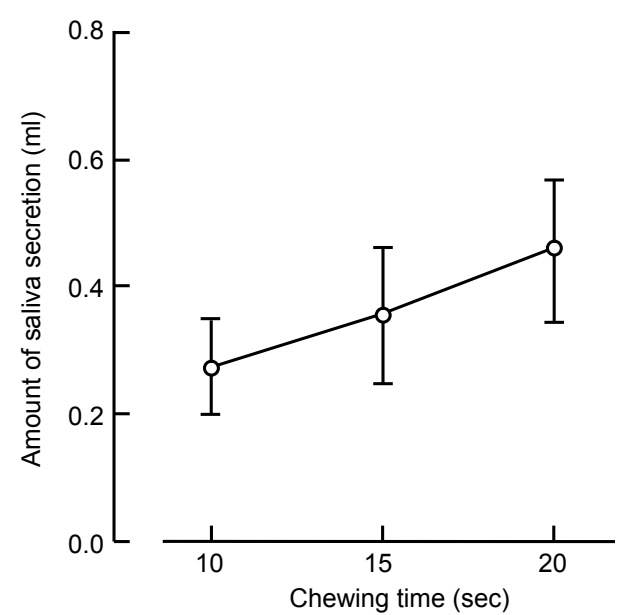

Fig.2 The average value and standard deviation of the amount of saliva secretion for 10, 15, and 20 seconds.

\section{Discussion}

Tanaka et $\mathrm{al}^{11}$ asked subjects to chew four types of cylindrical-shaped gummy jelly varying in size and weight on their habitual chewing side, and they found that when the length and diameter were $10 \mathrm{~mm}$ and the weight was $2 \mathrm{~g}$ the subjects were able to chew unconsciously without any stickiness or incompatibility and that this jelly was the most suitable test food. In addition, other results confirmed that masticatory movement was most stable when chewing gummy jelly containing $8 \%$ gelatin. ${ }^{15}$ Based on these reports, the gummy jelly used by Tanaka et $\mathrm{al}^{11}$ served as the test food.

Although a spectrophotometer, which requires only 20 to 25 minutes for recording and analysis, had previously been used to measure the amount of glucose extraction after chewing gummy jelly, ${ }^{11}$ it is costly, special agents are required for quantitative determination of color, and its use is relatively troublesome. In contrast, the portable blood glucose testing device used in this experiment only requires the patient to rinse his/her mouth out with $10 \mathrm{ml}$ of distilled water and place the test strip in the filtrate, and the glucose concentration would be automatically displayed after in 30 to 45 seconds. Additionally the portable blood glucose testing device was found to measure the amount of glucose extraction after chewing gummy jelly as accurately as a spectrophotometer after corrections are made. ${ }^{14}$ With the increase in the number of diabetic patients and the necessity to control the blood glucose lev-

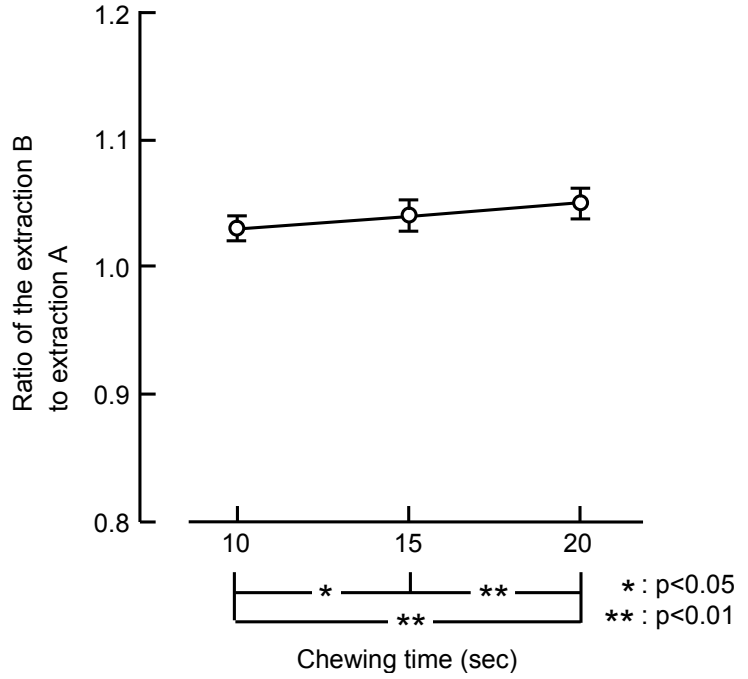

Fig.3 The average value and standard deviation of the ratio of the extraction $\mathrm{B}$ to extraction $\mathrm{A}$ for 10,15 , and 20 seconds.

els in these patients, relatively inexpensive and small, portable blood glucose testing devices have become available in the market, ${ }^{16-19}$ using which patients can easily measure their own blood glucose levels. Therefore in this experiment a portable blood glucose testing device was used to measure the amount of glucose extraction from chewing gummy jelly.

Tanaka et al $^{11}$ measured glucose extraction after chewing gummy jelly for 5, 10, 15, and 20 seconds, and investigated the relationship between the chewing time and the glucose extraction. They found that the glucose extraction increased in proportion to chewing time for up to 20 seconds. By measuring glucose extraction after chewing gummy jelly for 10, 20, and 30 times, Kobayashi et $\mathrm{al}^{13}$ investigated the relationship between the number of chewing strokes and the glucose extraction and found that the glucose extraction increased in proportion to chewing time. The result of this experiment measured the amount of glucose extraction after chewing gummy jelly for 10,15 , and 20 seconds showed that the glucose extraction increased in proportion to chewing time. Given that the cycle time during chewing of gummy jelly is about $600 \mathrm{msec},{ }^{11}$ chewing time can be surmised to be up to $20 \mathrm{sec}^{-}$ onds even with a maximum of 30 times, as was used by Kobayashi et al. ${ }^{13}$ Thus, the amount of glucose extraction during chewing of gummy jelly should be thought of as increasing proportional to time with a chewing time of up to 20 seconds, 
i.e. increasing proportional to chewing work.

During chewing for 10, 15, and 20 seconds, the amount of saliva secretion was respectively $0.28 \mathrm{ml}, 0.36 \mathrm{ml}$, and $0.46 \mathrm{ml}$. While the amount displays significant changes over time, it is, unlike the proportional changes displayed by the amount of glucose extraction, comparatively extensive during chewing for 10 seconds and increases afterwards in increments of about $0.1 \mathrm{ml}$ every 5 seconds. Assuming this trend continues for up to 1 minute, the amount of saliva secretion during chewing for 1 minute reaches about $1.2 \mathrm{ml}$, which is less than the $1.4-1.8 \mathrm{ml} / \mathrm{min} \mathrm{se}^{-}$ creted during chewing of paraffin wax. ${ }^{20,21}$ This is probably because paraffin wax with no moisture content requires more saliva during chewing than gummy jelly with moisture content of about $40 \%$. However, the reason for a relatively greater amount of saliva secretion during chewing for 10 seconds than with other chewing times is thought to be because, in addition to stimulation of oral sensation due to chewing, gummy jelly provides greater gustatory stimulus than other chewing times. Regardless, the amount of saliva secretion increased significantly as chewing of gummy jelly progressed, and the effect of this amount was about $3 \%$ during chewing for 10 seconds, about 4 $\%$ during chewing for 15 seconds, and about $5 \%$ during chewing for 20 seconds. Therefore, attention must be paid to the small amount of glucose extraction as is displayed because of the effects of saliva.

\section{Conclusion}

In order to clarify whether the amount of saliva secreted during chewing of gummy jelly affected the assessment of masticatory performance, 20 healthy subjects were asked to chew gummy jelly and the glucose extraction and saliva secretion after chewing were analyzed. It was concluded that the amount of saliva secretion increased during the chewing of gummy jelly and affected the assessment of masticatory performance, and the effect was less than $5 \%$ for chewing of up to 20 seconds.

\section{References}

1. Manly RS, Braley LC. Masticatory performance and efficiency. J Dent Res 29: 448-462, 1950.

2. Kaper KK, Yurkstas AA. Test foods for measuring masticatory performance of denture wearers. J Prosthet Dent 14: 483-491, 1964.

3. Hatch JP, Shinkai RSA, Sakai S et al. Determination of masticatory performance in dentate adults. Arch Oral Biol 46: 641-648, 2001.

4. Van der Bilt A, Van der Glass H, Olthoff LW et al. The effect of particle size reduction on the jaw gape in human mastication. J Dent Res 70: 931-937, 1991.

5. English JD, Buschang PH, Throckmorton GS. Does malocclusion affect masticatory performance? Angle Orthod 72: 21-27, 2002.

6. Al-Ali F, Heath MR, Wright PS. Simplified method of estimating masticatory performance. J Oral Rehabil 26: 678-683, 1999.

7. Hayakawa I, Watanabe I, Hirano S et al. A simple method for evaluating masticatory performance using a color-changeable chewing gum. Int J Prosthodont 11: 173-176, 1999.

8. Prinz JF. Quantitative evaluation of the effect of bolus size and number of chewing strokes on the intra-oral mixing of a two-color chewing gum. J Oral Rehabil 26: 243-247, 1999.

9. Anastassiadou V, Heath MR. The development of a simple objective test of mastication suitable for older people, using chewing gums. Gerodontology 18: 79-86, 2001.

10. Sato H, Fueki K, Sueda S et al. A new and simple method for evaluating masticatory function using newly developed artificial test food. J Oral Rehabil 30: 68-73, 2003.

11. Tanaka A, Shiga H, Kobayashi Y. Quantitative evaluation of mandibular movement and masticatory muscular activities by analyzing the amount of glucose discharge during gummy jelly chewing. J Jpn Prosthodont Soc 38: 1281-1294, 1994.

12. Okiyama S, Yoshida M, Nokubi T et al. Influence of physical properties of testing food and occlusal contact on masticatory efficiency. J Jpn Prosthodont Soc 40: 710-717, 1996.

13. Kobayashi Y, Shiga H, Arakawa I et al. The effectiveness of measuring glucose extraction for estimating masticatory performance. Prosthodont Res Pract 5: 104-108, 2006.

14. Shiga H, Kobayashi Y, Arakawa I et al. Validation of a portable blood glucose testing device in measuring masticatory performance. Prosthodont Res Pract 5: 15-20, 2006.

15. Kuriyama S, Shiga H, Kobayashi Y. Property of test food for masticatory movement analysis, the hardness of gummy jelly. J Jpn Prosthodont Soc 45: 592-601, 2001.

16. Conrad PD, Sparks JW, Osberg I et al. Clinical ap- 
plication of a new glucose analyzer in the neonatal intensive care unit: comparison with other methods. J Pediatrics 114: 281-287, 1989.

17. Edelman SV, Callahan P, Deeb LC. Multisite eval uation of a new diabetes self-test for glucose and glycated protein (fructosamine). Diabet Tech Ther 2: 233-238, 2000.

18. Tieszen KL, New JP. Alternate side blood glucose testing: do patients refer it? Diabet Med 20: 325-328, 2003.
19. Riddle MC. Measuring fructosamine at home. Diabet Tech Ther 2: 239-240, 2000.

20. Ericsson Y, Hardwick L. Individual diagnosis, prognosis and counselling for caries prevention. Caries Res 12 Suppl 1: 94-102, 1978.

21. Heintze U, Birkhed D, Bjorn H. Secretion rate and buffer effect of resting and stimulated whole saliva as a function of age and sex. Swed Dent J 7: 227-238, 1983. 